\title{
Methodological Considerations in the Analysis of Fecal Glucocorticoid Metabolites in Tufted Capuchins (Cebus apella)
}

\author{
Brandon C. Wheeler • Barbara Tiddi • \\ Urs Kalbitzer • Elisabetta Visalberghi • \\ Michael Heistermann
}

Received: 8 April 2013 / Accepted: 13 June 2013 /Published online: 16 August 2013

(C) The Author(s) 2013. This article is published with open access at Springerlink.com

\begin{abstract}
Analysis of fecal glucocorticoid (GC) metabolites has recently become the standard method to monitor adrenocortical activity in primates noninvasively. However, given variation in the production, metabolism, and excretion of GCs across species and even between sexes, there are no standard methods that are universally applicable. In particular, it is important to validate assays intended to measure GC production, test extraction and storage procedures, and consider the time course of GC metabolite excretion relative to the production and circulation of the native hormones. This study examines these four methodological aspects of fecal GC metabolite analysis in tufted capuchins (Cebus apella). Specifically, we conducted an adrenocorticotrophic hormone (ACTH) challenge on one male and one female capuchin to test the validity of four GC enzyme immunoassays (EIAs) and document the time course characterizing GC metabolite excretion in this species. In addition, we compare a common field-friendly technique for extracting fecal GC metabolites to an established laboratory extraction methodology and test for effects of storing "field extracts" for up to 1 yr. Results suggest that a corticosterone EIA is most sensitive to changes in GC production, provides reliable measures when extracted according to the field method, and measures GC
\end{abstract}

B. C. Wheeler $(\bowtie) \cdot$ B. Tiddi $\cdot$ U. Kalbitzer

Cognitive Ethology Laboratory, German Primate Center, 37077 Göttingen, Germany

e-mail: bcwheeler43@gmail.com

B. C. Wheeler · B. Tiddi • U. Kalbitzer

Courant Research Centre Evolution of Social Behaviour, University of Göttingen, 37077 Göttingen, Germany

E. Visalberghi

Unit of Cognitive Primatology, Institute of Cognitive Sciences and Technologies, National Research Council, Rome, Italy

M. Heistermann

Endocrinology Laboratory, German Primate Center, 37077 Göttingen, Germany 
metabolites which remain highly stable after even 12 mo of storage. Further, the time course of GC metabolite excretion is shorter than that described yet for any primate taxa. These results provide guidelines for studies of GCs in tufted capuchins, and underscore the importance of validating methods for fecal hormone analysis for each species of interest.

Keywords ACTH challenge - Captive welfare $\cdot$ Cortisol $\cdot$ Sapajus Storage effects · Stress

\section{Introduction}

Stressful events generally cause activity in the hypothalamic-pituitary-adrenal axis of vertebrate animals that eventually results in the production of glucocorticoid (GC) hormones (mainly cortisol and/or corticosterone, depending on the species) in the adrenal cortex (Sapolsky 2002). After their circulation in the blood, where the GCs target all major tissues of the body, the GCs are metabolized by the liver and kidneys and the metabolites are eventually eliminated from the body via excretion into the animal's urine and feces (Brownie 1992). Analysis of urinary and, in particular, fecal GC metabolites has become the standard method to measure adrenocortical activity noninvasively in a range of taxa, especially under wild conditions, but is also broadly applicable in studies of captive animal welfare (Anestis 2010; Boinski et al. 1999b; Keay et al. 2006). However, the production, metabolism, and excretion of GCs can differ between closely related species or even between sexes within a species (Bahr et al. 2000; Colby and Kitay 1972; Schwarzenberger et al. 1996; Touma et al. 2003; Wasser et al. 2000). The specific methods to be used to analyze fecal or urinary GC metabolites should thus be tested and validated for each species and sex of interest to ensure that results are biologically meaningful and appropriately interpreted (Goymann 2012; Heistermann et al. 2006; Hodges and Heistermann 2011; Palme 2005; Touma and Palme 2005; von der Ohe and Servheen 2002). Specifically, it is of particular importance to validate the assay used to quantify adrenocortical activity (Heistermann et al. 2006; Touma and Palme 2005), test the methods for extracting and storing hormone metabolites (Beehner and Whitten 2004; Hunt and Wasser 2003; Pappano et al. 2010; Shutt et al. 2012), and, depending on the question of interest, determine the time lag between hormone production and the excretion of the hormone metabolites (Schwarzenberger et al. 1996).

First, because GCs are metabolized by the liver and kidneys before excretion (Brownie 1992), only hormone metabolites, rather than the native hormone itself, can typically be reliably measured in the excreta (Touma and Palme 2005); assays that reliably quantify native GC concentrations in the blood of a given species may therefore provide unreliable measurements of adrenal activity when used to measure metabolites in the excreta of that same species (Heistermann et al. 2006; Shutt et al. 2012; Touma and Palme 2005). For example, although many studies examining fecal GC metabolites in primates have used assays designed to measure cortisol in the blood, some primate taxa excrete little native cortisol in the feces, causing the cortisol assay to be a relatively poor method for measuring GC output (Heistermann et al. 2006). In these cases, other assays, including those designed to measure 
corticosterone or reduced cortisol metabolites, may provide a better measure (Ganswindt et al. 2003; Heistermann et al. 2004, 2006; Möstl et al. 2002; Pirovino et al. 2011; Shutt et al. 2012). It is thus important to perform a physiological validation to confirm that a particular assay is able to measure adrenocortical function reliably and provide results that are biologically meaningful. The administration of exogenous adrenocorticotrophic hormone, i.e., ACTH challenge test, which causes an activation of adrenocortical activity and an increase in cortisol release from the adrenal cortex, is considered the gold standard in this regard (Heistermann et al. 2006; Touma and Palme 2005; Wasser et al. 2000; Whitten et al. 1998b).

Second, although keeping fecal samples frozen from collection until arrival at the laboratory for hormone analysis is considered ideal (Hodges and Heistermann 2011; Ziegler and Wittwer 2005), this is not always possible, especially under field conditions, owing to limitations in terms of infrastructure and transportation. Many field endocrinologists have thus opted for alternative storage methods, including extracting metabolites from fecal samples on site, that can be performed easily in the field and facilitate the transportation of samples from habitat countries to the lab (Ziegler and Wittwer 2005). However, comparisons of hormone metabolite concentrations based on such methods relative to fecal samples frozen until analysis are rarely made ( $c f$. Shutt et al. 2012). Further, the stability of hormone metabolites when using such field-friendly methods may vary among species (Kalbitzer and Heistermann 2013). It is therefore recommended to both validate the preservation method and test for storage effects based on that method before initiating field endocrinological studies with a given species (Kalbitzer and Heistermann 2013; Pappano et al. 2010; Pettitt et al. 2007; Shutt et al. 2012).

Finally, because hormone metabolites must pass through the digestive system before being excreted in feces, there is a temporal lag between the circulation of the hormones in the blood and the appearance of the hormone metabolites in the feces (Anestis 2010) that is largely a factor of gut passage rate (Schwarzenberger et al. 1996). Although this time lag tends to be between 20 and $48 \mathrm{~h}$ in most mammals (Anestis 2010; Heistermann et al. 2006; Palme et al. 1996; Schwarzenberger et al. 1996), it has been shown to be as little as $7 \mathrm{~h}$ in the common marmoset (Callithrix jacchus: Bahr et al. 2000; Heistermann et al. 2006) and $4 \mathrm{~h}$ in the common mouse (Mus musculus: Touma et al. 2003), likely resulting from relatively fast gut passage rates in these taxa (Caton et al. 1996; Karasov et al. 1986). In terms of assessment of stress physiology, this time lag in fecal hormone metabolite excretion results in a given sample tending to show average stress levels over a given period (Whitten et al. 1998a), facilitating analysis of chronic stressors but limiting the ability to determine the effects of acute events on endocrine activity, which can be better studied noninvasively through analysis of saliva or urine (Anestis 2010; Crockford et al. 2013; Surbeck et al. 2012; but see Edwards et al. 2013). Nevertheless, knowledge of the time course of hormone excretion can allow researchers to determine the timing of events related to hormone production, e.g., ovulation, with a certain degree of certainty (Charlton et al. 2010; Engelhardt et al. 2005; Higham et al. 2008).

This study examines these methodological aspects of fecal GC analysis in tufted capuchins (Cebus apella, taxonomically synonymous with Sapajus spp.), a species that has been relatively well studied under natural conditions and is among the most common primates used in captive behavioral and biomedical research (Fragaszy et al. 2004). Although at least three previous studies have examined GC production in 
tufted capuchins (Boinski et al. 1999a,b; Lynch et al. 2002; Ulyan et al. 2006) based on the use of cortisol assays in fecal material, the validity of this assay has thus far not been tested in tufted capuchins. Further, no previous studies have tested extraction procedures or storage effects or examined the time course characterizing the excretion of fecal GCs in this species.

Specifically, we first test the validity of four enzyme immunoassays (EIAs) that have previously been shown to measure adrenocortical activity accurately in various primate species of all major taxa, including cortisol- and corticosterone-specific assays as well as two group-specific assays that have been shown to measure reduced cortisol metabolites in primates (Fichtel et al. 2007; Heistermann et al. 2006; Martínez-Mota et al. 2008; Ostner et al. 2008; Pirovino et al. 2011; Shutt et al. 2012; Weingrill et al. 2011) and other vertebrates (Ganswindt et al. 2003; Möstl et al. 2002; Touma and Palme 2005). To test the ability of each of these assays to detect excreted GC metabolites in tufted capuchins, we collected fecal samples before and after the administration of ACTH and anesthetics, both of which have been well documented to increase GC production (Heintz et al. 2011; Heistermann et al. 2006; Touma and Palme 2005). By conducting this ACTH challenge with a captive male and female tufted capuchin during an annual health examination, we tested the relative suitability of the four assays to detect the physiologically induced increase in cortisol secretion and excretion of its fecal metabolites. In addition, we conducted high-performance liquid chromatography (HPLC) analysis on fecal extracts to assess the pattern of metabolites measured in the four assays and thus to characterize their specificity. Second, we describe the time course of GC metabolite excretion, i.e., the time from the ACTH-induced GC production until the peak concentration of GC metabolites in the feces and return to baseline levels. Third, we test a simple method for extracting the GC metabolites from wet feces that can be easily performed in the field and allows the samples to be stored in ethanol until transported to the lab for immunoassay. Fourth, we test for effects of storing the ethanolic fecal extracts for prolonged periods of time (up to $12 \mathrm{mo}$ ) on GC metabolite concentrations. Taken together, the results address a number of methodological considerations that should be taken into account in examining fecal GC and other steroid hormone metabolites in tufted capuchins.

\section{Methods}

Focal Individuals and ACTH Challenge

The subjects were one adult female (23 yr old) and one adult male (19 yr old) tufted capuchin housed in the Primate Center of the Istituto di Scienze e Tecnologie della Cognizione in Rome, Italy. Tufted capuchins, traditionally considered to comprise a single species, i.e., Cebus apella, throughout their biogeographical range, have more recently been divided into multiple species based on the phylogenetic species concept (Groves 2001; see also Lynch Alfaro et al. 2012 for details on an alternative genuslevel taxonomy). We chose to follow the traditional (one species) classification because the focal subjects' affinities to the multiple phylogenetic species are not clear and they are apparently hybrids of two or more of the phylogenetic species, 
suggesting that the traditional taxonomy would likely be retained under the biological species concept. Further, the latter seems a more relevant species concept for the purposes of describing species-specific biological patterns such as those investigated here.

The focal male and female subjects were members of two different social groups of five individuals housed in adjacent indoor-outdoor enclosures (indoor compartments: $4.45 \mathrm{~m}^{2}$ and $4.43 \mathrm{~m}^{2}$ for the male and female, respectively; outdoor compartments: $19.4 \mathrm{~m}^{2}$ and $38.7 \mathrm{~m}^{2}$ ) containing ropes, tree trunks, and a floor covered with wood chips for enrichment. The individuals are regular subjects of behavioral, physiological, and cognitive research (Addessi et al. 2007; Carosi et al. 1999; Sabbatini et al. 2012) and are monitored daily. The subjects were fed daily together with the other members of their respective groups with a diet of fruits and vegetables (including apples, pears, and onions) supplemented with standard monkey chow. Water was available ad libitum.

All subjects in the colony received their annual health examination by a veterinarian on October 29, 2010, which included the administration of $0.1 \mathrm{ml}$ of anesthetic (Imalgene 1000, Muriel, France) per kg body weight. Immediately after the anesthetic, the two focal individuals received an intramuscular injection of $0.1 \mathrm{ml}$ (10 IU) of synthetic ACTH (Synacthen, Biofutura Pharma, Italy). Although the peak of adrenocortical activity almost certainly occurred in association with each individual's own capture for the examination and the subsequent administration of the anesthetic and ACTH, which occurred within a few minutes of the capture, some degree of a stress reaction may have begun $c a$. $1.5 \mathrm{~h}$ before the male's examination and $2.5 \mathrm{~h}$ before the female's examination, when individuals housed in an adjacent enclosure were captured for the health examination. In any case, this circumstance might have affected only our determination of the lag time in GC response rather than the response pattern itself.

\section{Ethical Note}

To minimize the invasiveness of administration of the ACTH challenge, the method was applied to individuals already undergoing capture and anesthetization as part of a regular annual health examination, which is mandatory by law. Injections of synthetic ACTH are routinely used in veterinary practice to measure adrenocortical activity and have only minor, short-term effects. This study met the ethical standards of the Animal Behavior Society, was approved by the official veterinarian of the Primate Center of the Istituto di Scienze e Tecnologie della Cognizione, and was performed in accordance with European Law on humane care and use of laboratory animals.

\section{Sample Collection}

We collected two fecal samples per day from each of the two focal individuals in the mornings on the $4 \mathrm{~d}$ before the ACTH challenge (October 25-28, 2010) to establish baseline GC levels. On the day of the ACTH challenge, we collected all fecal samples voided from the two individuals after the injections until ca. 19:00. On the 2 following days (October 30-31), we collected approximately one fecal sample per individual per hour from 10:00 until 18:00. For the 3 final days (November 1-3), we collected one sample per individual per day during the morning. In total, we collected 
37 samples from the male and 32 from the female, all uncontaminated with urine. The subjects were kept with one other individual from their group or alone in the indoor compartment of their enclosures for sample collection; in cases in which the subjects were kept with another individual from their social group, we collected samples only if the subject of interest was directly observed to have defecated. During the $2 \mathrm{~d}$ after the checkup in which approximately one sample per hour was collected, the subjects were allowed to rejoin their group in the outdoor enclosure each hour after collection of a sample, and were then moved back into the indoor enclosure alone or with one group mate for the collection of the next sample. All samples were stored in polypropylene tubes and frozen at $-15^{\circ} \mathrm{C}$ within 30 min of voiding. Samples were then transported to the Endocrinology Laboratory at the German Primate Center on dry ice and kept frozen until further processing.

\section{Hormone Extraction}

We performed extractions of GC metabolites from all fecal samples using two methods. First, we extracted metabolites from thawed samples based on a "field extraction method" of wet feces similar to the method described in Palme (2005) but with some minor modifications. Specifically, we removed and weighed between 0.4 and $0.6 \mathrm{~g}$ of homogenized wet feces from the sample (in two cases, the weight was slightly lower, 0.35 and $0.22 \mathrm{~g}$, because the samples were relatively small). We then added $5 \mathrm{ml}$ of $80 \%$ ethanol (for the two samples in which a smaller amount of wet feces was taken, we added only 3.5 and $2.2 \mathrm{ml}$, respectively, to keep the approximate ratio of fecal mass to ethanol volume consistent) and vortex-mixed the samples for $5 \mathrm{~min}$. Samples were then centrifuged at $2000 \mathrm{rpm}$ for $10 \mathrm{~min}$ and $1 \mathrm{ml}$ of the supernatant was removed and stored in a 2-ml polypropylene tube at $-20{ }^{\circ} \mathrm{C}$ until hormone assays were performed (see Kalbitzer and Heistermann 2013; Ziegler and Wittwer 2005 for an explanation of how such methods can be performed with basic equipment in a field laboratory). The fecal pellet of each sample was dried to a constant weight after which the dry weights were determined.

Second, we extracted the remaining portion of each fecal sample according to a standard "lab method" described in Heistermann et al. (1995) and that is often used in fecal hormone analysis. In short, we lyophilized the fecal material, pulverized it with a mortar and pestle, and then sieved the powder through a fine wire mesh. We weighed 30-70 mg of the sieved powder from each sample (only 9.6, 13.8, and $26.4 \mathrm{mg}$ in three cases in which samples were small), placed it into a polypropylene tube, and extracted the GC metabolites with $3 \mathrm{ml}$ of $80 \%$ methanol (1 $\mathrm{ml}$ in the three cases of smaller weights) by vortex-mixing for $10 \mathrm{~min}$. Samples were then centrifuged at $3000 \mathrm{rpm}$ for $10 \mathrm{~min}$ and the supernatant was removed and stored at $-20{ }^{\circ} \mathrm{C}$ until hormone assays were performed.

\section{Enzyme Immunoassay and HPLC Analysis}

Samples extracted according to the lab extraction method were analyzed with four different EIAs that have been shown to differ in their specificity of measuring GC metabolites, including a cortisol (CORT; Palme and Möstl, 1997), corticosterone (CCST; Heistermann et al. 2006), and two group-specific assays measuring 5ß- 
reduced cortisol metabolites with a $3 \alpha, 11$-oxo ( $3 \alpha, 11$-oxo-CM; Möstl et al. 2002) or $3 \alpha, 11 \beta$-dihydroxy structure ( $3 \alpha, 11 \beta$-dihydroxy-CM; Ganswindt et al. 2003). All four assays have been used successfully to monitor GC output in various other primate and nonprimate species (Fichtel et al. 2007; Ganswindt et al. 2003; Heistermann et al. 2004, 2006; Martínez-Mota et al. 2008; Pirovino et al. 2011; Wasser et al. 2000).

We performed all hormone assays on microtiter plates as described in detail by Heistermann et al. (2004, 2006). Information on antibody characteristics, standards, and labels used as well as on other assay details, e.g., data on assay sensitivities, is given in Heistermann et al. (2006). Intra- and interassay coefficients of variation of high- and low-value quality controls for each assay were $<10 \%$ and $<13 \%$, respectively. Hormone concentrations are usually expressed as $\mu \mathrm{g} / \mathrm{g}$ dry fecal weight, except for HPLC data, which are reported as ng/fraction.

To assess the pattern of metabolites measured and thus characterize the specificity of the four GC assays, we performed reverse-phase high performance liquid chromatography (RP-HPLC) on the methanolic fecal extracts of the study male and female representing peak GC response to the ACTH challenge using the procedure previously described by Möhle et al. (2002) and Heistermann et al. (2006). The HPLC system used also allowed us to evaluate whether the GC assays tested show a co-measurement of certain fecal androgens that may potentially be detected by antibodies raised against GC metabolites (Ganswindt et al. 2003; Heistermann et al. 2006; Möstl et al. 2002). After HPLC, we measured each fraction in all four $\mathrm{GC}$ assays to generate the profiles of immunoreactivity.

\section{Test of Storage Effects}

To evaluate whether long-term storage of capuchin fecal extracts in a refrigerator (4$7{ }^{\circ} \mathrm{C}$ ) or a freezer $\left(-20^{\circ} \mathrm{C}\right)$ results in changes in $\mathrm{GC}$ metabolite concentrations, we conducted a storage experiment. For this, we selected 16 extracts generated by the field extraction method from the two focal individuals ( 8 from each of the male and the female) and stored them for up to $1 \mathrm{yr}$ in the two aforementioned conditions. We measured samples in the CCST and $3 \alpha, 11 \beta$-dihydroxy-CM assays only, because these two assays provided strong responses to the ACTH challenge in both sexes and were thus considered most suitable for monitoring adrenocortical activity in the study species. We measured GC levels with the two assays within 2 wk after extraction to provide control values, i.e., 0 mo of storage, and then after 3, 6, 9, and 12 mo of storage. We compared GC concentrations measured after the respective storage durations to those of the control measurement to determine if levels have changed over time. In addition, because samples stored at $-20{ }^{\circ} \mathrm{C}$ seem to be highly stable (Kalbitzer and Heistermann 2013; Shutt et al. 2012), we also made comparisons between refrigerated and frozen samples for each time point. Because refrigerated and frozen samples for a given storage time and a given EIA were measured on the same assay plate, direct comparisons of GC concentrations for the two storage methods can provide some insight into the degree to which any observed differences from the controls are explained by interassay variation. For this experiment, interassay coefficients of variation of a high- and low-value quality control were $4.1 \%$ and $7.5 \%$ for the CCST assay and $7.2 \%$ and $8.8 \%$ for the $3 \alpha, 11 \beta$-dihydroxyCM assay. 


\section{Data Analysis}

We assessed physiological validation of each of the EIAs by simple graphical analysis of the percent change in GC metabolite concentrations in posttreatment samples relative to the mean of pretreatment (baseline) values. Validation was demonstrated by the EIAs that produced temporal profiles showing a clear peak in GC metabolite concentrations and a subsequent return to baseline values after administration of the ACTH. Similarly, the time course of GC metabolite excretion is described based on the time from the administration of the anesthetic and ACTH to the first detected rise in GC metabolite concentrations, their peak, and return to baseline levels. We defined "baseline levels" as GC metabolite concentrations equal to or less than 2 standard deviations of the mean of all baseline samples collected for that individual. We tested the reliability of hormone metabolite measurements obtained with the field extraction method by comparing those values to those obtained using the lab extraction method with a Spearman rank correlation. Finally, to determine if samples extracted according to the field method demonstrated storage effects after 3, 6, 9, or 12 mo, we first conducted Wilcoxon signed-ranks exact tests by considering the percent change in GC metabolite concentrations from the controls for each of the four time points, two storage conditions (refrigerator and freezer), and for each of the two assays considered. We also used Wilcoxon signedranks exact tests to test for differences between the two storage conditions for each time point and assay. Although this resulted in a large number of individual tests (24), we opted to not correct for multiple testing, as this would increase the likelihood of a type II error and thus suggest no storage effect in cases in which storage effects might indeed be present; we thus consider this a more cautious approach in the present case. In addition, because significant differences between samples measured on different plates can be due to simple interassay variation rather than true storage effects (Shutt et al. 2012), we considered differences from control values that were similar to the variation observed in the quality control measures to most likely be an artifact of interassay variation, even if differences were statistically significant.

\section{Results}

Physiological Validation and HPLC Analysis

All four EIAs detected a strong increase in fecal GC metabolite concentrations after the administration of the ACTH challenge and anesthetic, although they differed considerably in their sensitivity to the resultant increase (see Table I for baseline and peak concentrations for each assay and study subject). The CCST assay showed the most marked elevation in both the male and the female, while the CORT assay showed the weakest elevation in both sexes (Fig. 1). The two group-specific assays were intermediate in their sensitivity to detecting the increase, with the $3 \alpha, 11 \beta$-dihydroxy-CM assay being the more sensitive of the two in the female (Fig. 1a), and the $3 \alpha, 11$-oxo-CM responding better in the male (Fig. 1b). A similar pattern emerged on the third and fourth days after the ACTH challenge, when all assays except the CORT assay showed an increase in immunoreactive GC metabolite levels over the values of the 2 previous days (Fig. 1). It is unclear, however, what caused this increase, as there was no observed 
Table I Baseline and peak concentrations of GC metabolites and the time course of their excretion in the feces of a male and female tufted capuchin

\begin{tabular}{|c|c|c|c|c|c|}
\hline & & CCST & CORT & $3 \alpha, 11 \beta$-Dihydroxy-CM & $3 \alpha, 11-$-ххо-CM \\
\hline \multirow[t]{4}{*}{ Female } & Baseline $(\mu \mathrm{g} / \mathrm{g} \text { feces })^{\mathrm{a}}$ & $2.28 \pm 2.25$ & $11.76 \pm 5.79$ & $0.21 \pm 0.25$ & $1.57 \pm 2.18$ \\
\hline & Peak $(\mu \mathrm{g} / \mathrm{g} \text { dry feces })^{\mathrm{b}}$ & 68.59 & 104.30 & 4.50 & 22.69 \\
\hline & Time to peak $(\mathrm{h})^{\mathrm{c}}$ & 2.1 & 2.1 & 2.1 & 2.1 \\
\hline & Return to baseline $(\mathrm{h})^{\mathrm{d}}$ & $5.0-6.3$ & $7.1-22.5$ & $4.0-5.0$ & $5.0-6.3$ \\
\hline \multirow[t]{4}{*}{ Male } & Baseline ( $\mu \mathrm{g} / \mathrm{g}$ feces) & $0.20 \pm 0.13$ & $3.96 \pm 3.48$ & $0.12 \pm 0.07$ & $0.30 \pm 0.17$ \\
\hline & Peak ( $\mu \mathrm{g} / \mathrm{g}$ dry feces) & 61.10 & 138.20 & 9.87 & 53.53 \\
\hline & Time to peak (h) & 3.0 & 3.0 & 3.0 & 3.0 \\
\hline & Return to baseline (h) & $7.8-8.4$ & $8.4-23.8$ & $4.5-5.0$ & $5.0-5.5$ \\
\hline
\end{tabular}

\footnotetext{
${ }^{\text {a }}$ Values shown are mean $\pm \mathrm{SD}$ of all samples collected before the health examination

${ }^{\mathrm{b}}$ Highest measured concentrations of GC metabolites after administration of the ACTH and anesthetic

${ }^{\mathrm{c}}$ Time from the administration of the ACTH and anesthetic to defecation of the sample with the highest GC metabolite measures. For both individuals this was the first sample voided by the focal individual after reawakening from the health examination

${ }^{\mathrm{d}}$ Time from the administration of the ACTH and anesthetic to return to baseline levels. The first number shown is the time to the last sample that was $>2$ SDs of the mean of baseline samples, while the second is the time to the first sample at or below that level
}

stressful event that would predict such an elevation in both animals. Although it is possible that this was an effect of being separated from other group members, such an effect would be expected to have been even stronger in the previous days because the period of separation had been considerably longer.

The HPLC analysis revealed similar patterns of immunoreactivity in the female and the male, but variation between the different assays in both the levels of immunoreactivity and the fractions at which immunoreactivity was detected (Fig. 2). The highest level of immunoreactivity in both the female and the male was measured by the CORT assay, with a single peak occurring at fraction 14/15, respectively (the positions of native cortisol; Fig. 2). CCST immunoreactivity was also high, with both sexes evincing a single peak at fraction 22, where native corticosterone elutes. The two group-specific assays demonstrated considerably lower levels of immunoreactivity, which, in contrast to the immunoreactivity profiles obtained for CORT and CCST, were associated with several peaks occurring between fractions 9 and 38 for both assays (Fig. 2). In each assay, immunoreactivity measured beyond fraction 40, where major androgen metabolites elute in our HPLC system, was negligible.

\section{Time Course of GC Metabolite Excretion}

For both the female and the male, the peak response in fecal GC metabolite levels (which would be expected to reflect the adrenocortical activity related to the ACTH and anesthetic rather than to potentially stressful cues preceding the focal subjects' capture, including the capture of neighboring groups) occurred in the first sample voided by the study subjects after awakening from the anesthetic administered during the health examination. This occurred 2.1 and $3.0 \mathrm{~h}$ after the administration of the 

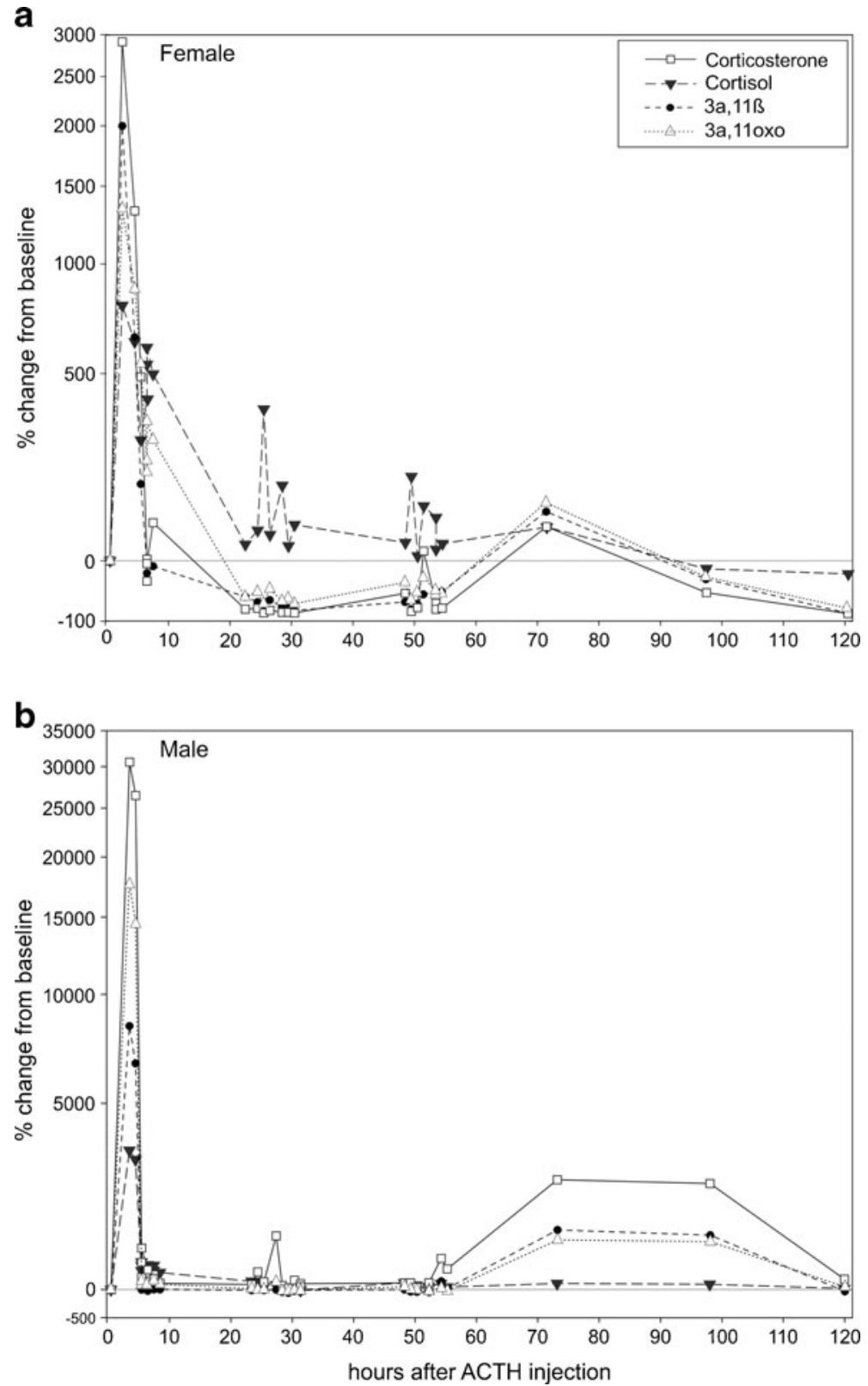

Fig. 1 Temporal profiles of immunoreactive GC metabolite concentrations measured in each of the four EIAs tested in the study for the (a) female and (b) male tufted capuchin housed in the Primate Center of the Istituto di Scienze e Tecnologie della Cognizione in Rome, Italy. The percent difference from baseline levels is calculated based on the mean values of samples collected before administration of the ACTH challenge.

ACTH and anesthetic in the female and the male, respectively (Table I). In both individuals, the GC metabolite levels as measured by three of the four assays returned to baseline levels by $4.0-8.4 \mathrm{~h}$ after the challenge test (Table I). Metabolites measured 

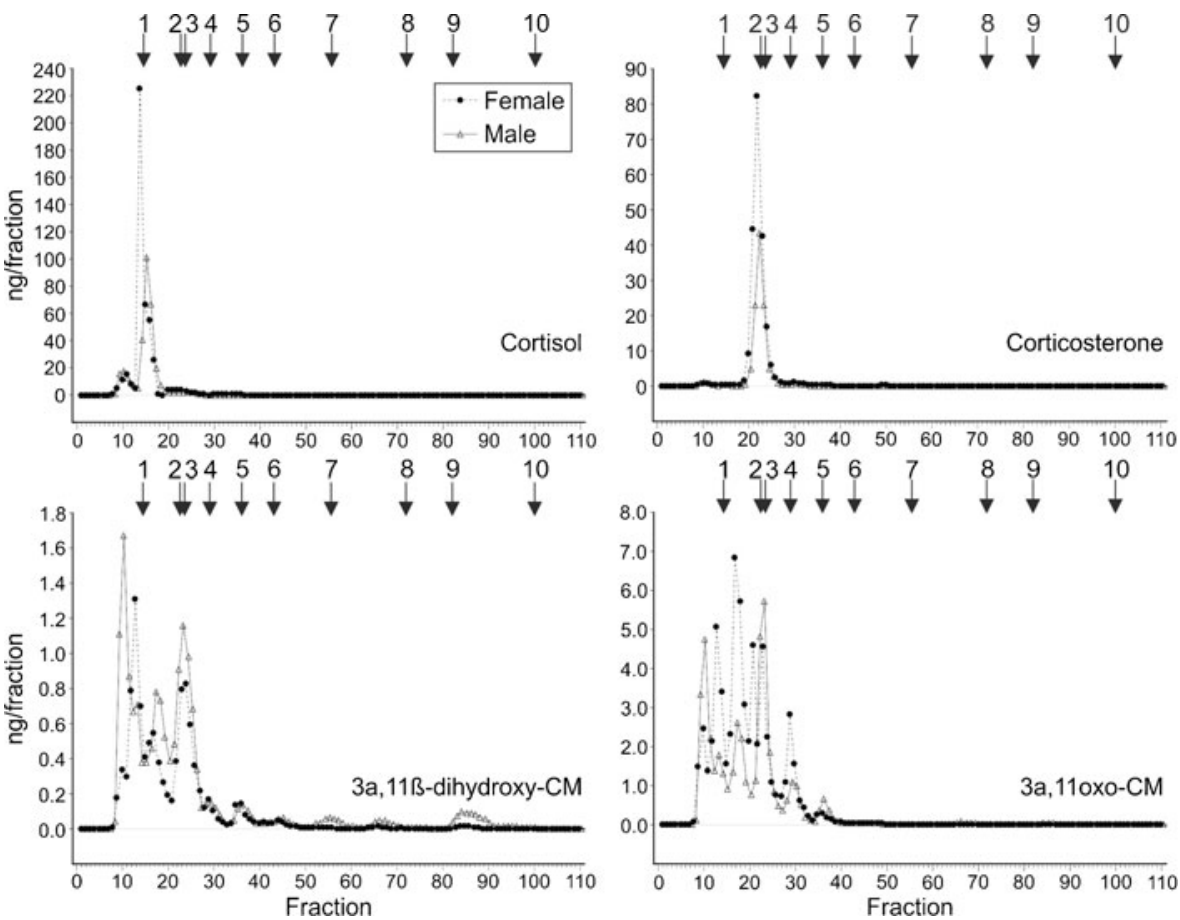

Fig. 2 HPLC profiles of immunoreactivity detected with the each of the four EIAs tested in this study for a male and a female tufted capuchin housed in the Primate Center of the Istituto di Scienze e Tecnologie della Cognizione in Rome, Italy. Samples tested were those that showed peak GC metabolite concentrations after the ACTH challenge. Arrows and numbers show the location of associate elution positions of reference standards: 1) cortisol (fractions 14-15), 2) corticosterone (22), 3) 11ß-hydroxyetiocholanolone (24), 4) 11oxoetiocholanolone $(29)$, 5) $5 \beta$-androstane-3,11,17-trione $(36), 6)$ testosterone $(43)$, 7) androstendione, dehydroepiandrosterone $(55-56), 8)$ epiandrosterone, $5 \beta$-DHT, 5b-androstane-3 $\beta$-ol-17-one (72), 9) $5 \beta$ androstane- $3 \alpha$-ol-17-one (82), and 10) androsterone (100).

with the CORT assay were still slightly above baseline levels in the last sample collected on the day of the health examination (at 7.1 and $8.4 \mathrm{~h}$ in the female and male, respectively), but were again at baseline levels in the first sample collected on the following day (Table I). Metabolites measured with the CORT assay were marginally higher than baseline in some, but not all, of the female's samples for up to $51.9 \mathrm{~h}$ after the health examination. The extent to which this was a consequence of the ACTH and anesthetic is not clear; alternatively, while this conceivably could have been a reaction to spending much of the day separated from other group members, the fact that the more sensitive assays showed no such response makes this an unlikely explanation.

Test of Field Extraction Method

Concentrations of fecal GC metabolites based on the field extraction method correlated strongly with those based on the lab extraction method for both of the assays used in this test, although the correlation was slightly stronger for samples measured with the CCST assay (Spearman's rank correlation: $r=0.947, N=58, P<0.001)$ than those measured with the $3 \alpha, 11 \beta$-dihydroxy assay $(r=0.885, N=61, P<0.001)$. 


\section{Test of Storage Effects}

Storage tests revealed some differences in the stability of fecal GC metabolite concentrations over time, based on both the storage method, i.e., refrigerator vs. freezer, and the metabolites measured. Values obtained with the CCST assay did not differ based on storage method and showed minimal differences based on storage time (Table II; Fig. 3a). In terms of storage time, the only significant differences found were between control values and samples stored frozen for 3 mo and samples stored under both conditions after 9 mo; samples stored frozen did not differ significantly after 6 or 12 mo, nor were there any differences between controls and refrigerated samples after 3, 6, or 12 mo (Table II, Fig. 3a). Further, even in cases in which the differences from the controls were significant, the mean percent difference from the controls (frozen condition at 3 mo: $8.7 \%$; frozen condition at 9 mo: $11.6 \%$; refrigerated condition at 9 mo: $10.2 \%$ ) was similar to the variation seen in the quality control measurements, i.e., interassay variation: $7.5 \%$.

Metabolites measured with the $3 \alpha, 11 \beta$-dihydroxy assay showed considerably more variation both over time and between the two storage methods (Table II; Fig. 3b). GC metabolite concentrations in samples stored in the refrigerator differed from those stored frozen after 3, 9, and 12 mo (Table II; Fig. 3b). Although samples stored in both the refrigerator and the freezer did not differ from controls after 3 or 6 mo, samples stored under both conditions differed significantly from controls after 9 and 12 mo when levels were elevated by $11.2-17.2 \%$ in the freezer condition and $c a$. $30 \%$ in the refrigerator condition (Table II; Fig. 3b). The magnitude of this variation

Table II Results of the Wilcoxon signed-ranks exact tests, testing for potential temporal storage effects on fecal GC metabolites concentrations in tufted capuchins

\begin{tabular}{|c|c|c|c|c|c|c|c|}
\hline Comparison & $T$ & $N$ & $P$ & Comparison & $T$ & $N$ & $P$ \\
\hline $\mathrm{R}-\mathrm{C}(\mathrm{CCST})$ & & & & $\mathrm{R}-\mathrm{C}(3 \alpha, 11 \beta)$ & & & \\
\hline $3 \mathrm{mo}$ & 40 & 16 & $>0.10$ & $3 \mathrm{mo}$ & 44 & 16 & $>0.10$ \\
\hline $6 \mathrm{mo}$ & 47 & 16 & $>0.10$ & $6 \mathrm{mo}$ & 39 & 16 & $>0.10$ \\
\hline $9 \mathrm{mo}$ & 27 & 16 & $<0.05$ & $9 \mathrm{mo}$ & 7 & 16 & $<0.01$ \\
\hline $12 \mathrm{mo}$ & 34 & 16 & $>0.05$ & $12 \mathrm{mo}$ & 6 & 16 & $<0.01$ \\
\hline $\mathrm{F}-\mathrm{C}(\mathrm{CCST})$ & & & & $\mathrm{F}-\mathrm{C}(3 \alpha, 11 \beta)$ & & & \\
\hline $3 \mathrm{mo}$ & 16 & 16 & $<0.01$ & $3 \mathrm{mo}$ & 41 & 14 & $>0.10$ \\
\hline $6 \mathrm{mo}$ & 37 & 15 & $>0.10$ & $6 \mathrm{mo}$ & 46 & 15 & $>0.10$ \\
\hline $9 \mathrm{mo}$ & 4 & 16 & $<0.01$ & $9 \mathrm{mo}$ & 22 & 16 & $<0.05$ \\
\hline $12 \mathrm{mo}$ & 28 & 15 & $>0.05$ & $12 \mathrm{mo}$ & 23 & 16 & $<0.05$ \\
\hline $\mathrm{R}-\mathrm{F}(\mathrm{CCST})$ & & & & $\mathrm{R}-\mathrm{F}(3 \alpha, 11 \beta)$ & & & \\
\hline $3 \mathrm{mo}$ & 67 & 16 & $>0.10$ & $3 \mathrm{mo}$ & 16 & 14 & $<0.05$ \\
\hline $6 \mathrm{mo}$ & 64 & 16 & $>0.10$ & $6 \mathrm{mo}$ & 30 & 16 & $>0.05$ \\
\hline 9 mo & 65 & 16 & $>0.10$ & 9 mo & 4 & 16 & $<0.01$ \\
\hline $12 \mathrm{mo}$ & 44 & 15 & $>0.10$ & $12 \mathrm{mo}$ & 3 & 14 & $<0.01$ \\
\hline
\end{tabular}

$P>0.05$ indicates $0.05<P<0.10 . \mathrm{R}-\mathrm{C}=$ refrigerator-control comparison; $\mathrm{F}-\mathrm{C}=$ freezer-control comparison; $\mathrm{R}-\mathrm{F}=$ refrigerator - freezer comparison 


\section{a}

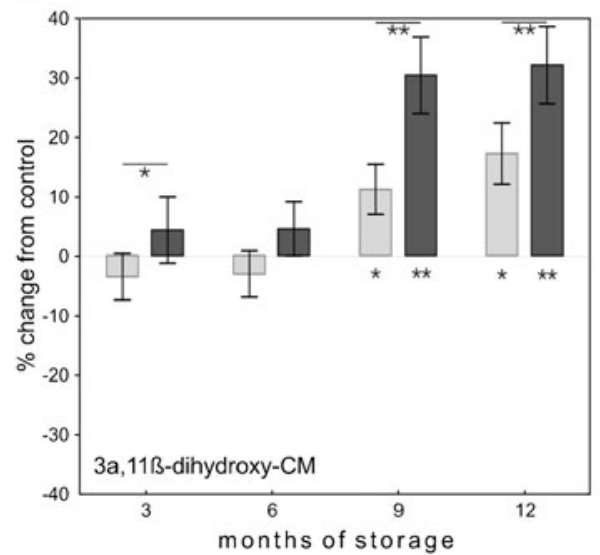

b

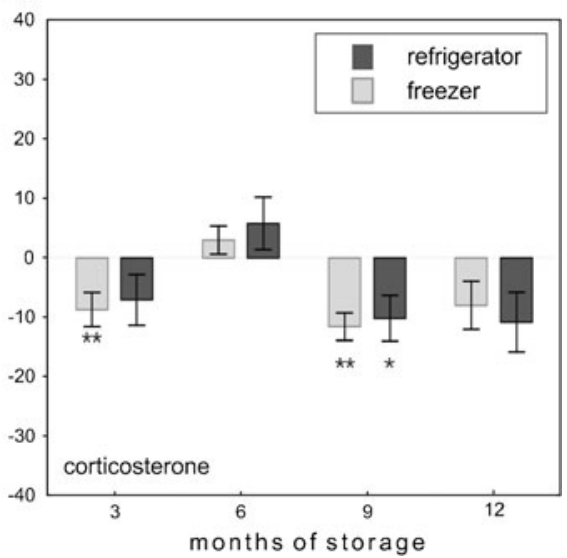

Fig. 3 Mean percent change ( \pm SE) from control samples in GC metabolite concentrations measured by each of the (a) $3 \alpha, 11 \beta$-dihydroxy and (b) corticosterone EIA after 3, 6, 9, and 12 mo of storage in both the frozen $\left(-20^{\circ} \mathrm{C}\right)$ and refrigerated condition. Samples were obtained from one male and one female tufted capuchin housed in the Primate Center of the Istituto di Scienze e Tecnologie della Cognizione in Rome, Italy. Significant differences from controls as well as between the two conditions are shown. Note that differences of $<13 \%$ fall into the range of interassay variation as measured by variation in quality controls. Asterisks above the bars indicate significant differences between refrigerated and frozen samples, while those below the bars indicate significant differences from controls. $* P<0.05, * * P<0.01$. See Table II for more details on the results of the statistical analyses.

exceeded that expected from simple interassay variation, particularly in the refrigerated samples.

\section{Discussion}

Tests of physiological validity and HPLC analysis indicate that all four glucocorticoid EIAs tested are suitable for the detection of changes in adrenocortical activity in tufted capuchins through analysis of fecal GC metabolites. First, each of the EIAs detected the physiological response via an increase in GC metabolite concentrations and a subsequent return to baseline values after administration of the ACTH and anesthetic. Second, the HPLC analysis indicated that in all four EIAs immunoreactivity was found exclusively at positions known for other primates to represent positions where cortisol metabolites elute (Heistermann et al. 2006) and that none of the EIAs demonstrate major cross-reactivity with androgens that potentially can have distorting effects on GC results (Ganswindt et al. 2003; Goymann 2012; Schatz and Palme 2001).

Although each of the EIAs thus appears suitable for fecal GC analysis in this species, there were differences in their sensitivity to the increase in GC production, with the CCST being the most sensitive, i.e., showing the strongest response to the ACTH and anesthetic, the two group-specific assays being intermediate, and the CORT assay being the least sensitive. This result was somewhat unexpected because, in many other species of primates and other mammals, measurement of native hormones in feces does not provide the most sensitive index of adrenocortical 
activity, whereas the measurement of reduced cortisol metabolites using groupspecific assays has been shown to be generally more reliable in this respect (Fichtel et al. 2007; Heistermann et al. 2006; Möstl et al. 2002; Pirovino et al. 2011; Weingrill et al. 2011). In addition, both native CCST and CORT appeared to be quantitatively most abundant in the feces of capuchins, exceeding levels of group-specific metabolites substantially, a finding also in marked contrast to that reported for most other mammalian species in which the two native hormones are usually present in feces in only very small amounts (Fichtel et al. 2007; Heistermann et al. 2006; Möstl et al. 2002; Pirovino et al. 2011). Thus, our data confirm previous findings of differences among primate species in the metabolism and excretion of glucocorticoids (Bahr et al. 2000; Heistermann et al. 2006) and emphasize that a priori predictions about the relative abundance of glucocorticoids in the feces of a given species cannot be made reliably. Our finding that the most abundant compound, immunoreactive CORT, showed the lowest response to adrenocortical stimulation is somewhat unexpected. The most likely explanation for this finding is that baseline concentrations measured by the CORT assay were markedly higher (7- to 60-fold) than those measured by the three other assays (see Table I). As a result, the relative increase in CORT levels in response to the ACTH challenge was less pronounced compared to the other three measures, although in terms of absolute levels (and in accordance with the HPLC data) CORT showed the highest peak value concentrations after adrenocortical stimulation (Table I). Our data thus show that measurement of the most abundant immunoreactive glucocorticoid in feces is not necessarily the most sensitive method for quantifying variation in adrenocortical activity (see also Heistermann et al. 2006; Table 1 in Young et al. 2004). This underscores the importance of testing different GC assays as part of a validation process, as making assumptions about the relative value of a single assay in terms of reflecting GC output reliably in a given species can be misleading. Nevertheless, despite the relative weakness of the CORT assay compared to the other three EIAs tested, our results indicate that results stemming from prior studies that used a CORT assay to measure fecal GC metabolites in tufted capuchins (Boinski et al. 1999a,b; Lynch et al. 2002; Ulyan et al. 2006) were likely biologically meaningful, although additional tests of extraction methods and storage effects for these metabolites would be ideal.

The results of the comparison of GC metabolite concentrations between the standard laboratory and the field method suggest that the latter allows for reliable measurement of adrenocortical activity in tufted capuchins, as has been previously demonstrated in gorillas (Gorilla gorilla: Shutt et al. 2012), with values obtained from assaying samples using the two extraction methods producing values that correlated highly with one another. Further, GC metabolites extracted according to the field method and measured with the CCST assay remained stable for $\geq 12$ mo at both subzero and refrigerator temperatures. Although samples differed significantly from control values after 3 and 9 mo, the size of the difference was small (ca. 10\%) and similar to the variation seen in the quality controls, suggesting that the variation in GC metabolite concentrations measured with the CCST over time is better explained by interassay variation (Kalbitzer and Heistermann 2013; Shutt et al. 2012) than by true degradation of the metabolites over time.

In contrast to metabolites measured with the CCST EIA, samples measured with the $3 \alpha, 11 \beta$-dihydroxy-CM EIA demonstrated high levels of stability for only 6 mo, after 
which GC metabolite concentrations increased significantly, and, at least for refrigerated samples, the amount of change from the controls exceeded that expected from interassay variation. This rise in $3 \alpha, 11 \beta$-dihydroxy-CM concentrations after 6 mo of storage cannot be explained by simple evaporation of the alcohol because this would be predicted to have affected the CCST measurements in the same way (because the same extract was measured with both EIAs), which was not the case. Thus, in contrast to findings in other studies (Kalbitzer and Heistermann 2013; Shutt et al. 2012), our data would suggest a time-dependent change in the structure or composition of the metabolites measured with our group-specific antibody. The fact that the extent of the increase in $3 \alpha, 11 \beta$-dihydroxy-CM levels was more pronounced at higher temperature would be in line with such an assumption (Khan et al. 2002). HPLC analysis and generation of immunoreactivity profiles of samples at time 0 and at different times of storage are needed, however, to substantiate this idea (cf. Möstl et al. 1999).

Taking together the results of the validation experiment, the HPLC analysis, and the storage tests, the CCST assay appears to be the best suited of the four for assessing adrenocortical activity based on analysis of fecal GC metabolites in tufted capuchins. This is especially the case for field studies in which GC metabolites are extracted in the field and stored for extended periods as ethanolic extracts. Further, these results provide additional evidence that ethanolic extracts can be stored at temperatures above freezing for $\geq 6$ mo without creating major storage effects (Kalbitzer and Heistermann 2013; Shutt et al. 2012), suggesting this as a promising storage method across taxa.

The very short time lag between the physiological stimulation of HPA axis activity and the appearance of peak GC metabolite concentrations in the feces (as little as $2 \mathrm{~h}$ ) and rapid return to baseline levels is, as far as we know, the shortest time course for fecal hormones thus far demonstrated in any mammalian species, shorter even than the 4- to 6-h lag demonstrated in the common mouse (Touma et al. 2003). Previous comparisons between urinary and fecal hormone metabolites of capuchins from the same study colony suggested a similarly short time course for female reproductive hormones (Carosi et al. 1999). The reason for such a short time course likely results from the very fast gut-passage rate characteristic of capuchin species (Milton 1984; Wehncke et al. 2003). Although gut transit times can differ based on specific food items eaten (Caton et al. 1996) and may thus potentially differ between wild and captive subjects, tufted capuchins (Cebus apella nigritus) in wild settings also demonstrate gut passage rates as fast as 1.75-3 $\mathrm{h}$ (Wheeler and Tiddi, unpubl. data). Such rapid gut passage may be attributable to the unusually small cecum of capuchins relative to other primates and most other mammalian taxa (although it is also characteristic of humans), and is thought to be an adaptation for feeding on foods that are easily digested (Martin 1990). Although a number of factors beyond diet contribute to interspecific differences in gut transit times, it would be worth testing the hypothesis that frugivorous primates will tend to show a faster time course for the excretion of fecal hormone metabolites than will folivores, owing to the faster gut transit times typical of the former (Lambert 1998).

Importantly, this short time course, similar to that observed to characterize the excretion of urinary hormone metabolites in this and other species (Anestis 2010; Brown et al. 2005; Carosi et al. 1999), suggests that investigation of the consequences of acute stressors on adrenocortical activity through analysis of fecal GC 
metabolites may be conducted more easily in tufted capuchins than in most other primate taxa. For example, by matching samples to detailed behavioral observations, it may be possible to test for the effects of affiliation, competition, e.g., for food or mates, or aggression on stress levels based on fecal samples collected in the hours after the event (Edwards et al. 2013; see also Surbeck et al. 2012 for similar analyses based on urinary GC metabolites). Although the temporal window for collecting fecal samples associated with such events appears to be generally quite short, it is possible that this window is somewhat extended for GC metabolites measured with the CORT assay (see Table I), although peak levels of these metabolites occur within the same time frame as the metabolites measured with the other three assays.

Finally, although small sample sizes are typical of fecal hormone validation experiments conducted with wildlife generally and nonhuman primates specifically (e.g., Table 1 in Palme et al. 2005), the sample size in the current study $(N=2$ individuals) limits the ability to conclude firmly that the current results represent species-specific patterns, especially concerning the assay validation and time course of excretion. However, very similar profiles resulted from samples collected in association with an ACTH challenge and anesthetic (using the same methods as the current study) conducted on the same male and a second female during the previous year's (2009) health examination. Detailed results from that experiment are not presented here because the unprocessed samples were unintentionally thawed and left at room temperature for $\geq 2 \mathrm{wk}$, and are thus unlikely to provide measures directly comparable to those obtained from samples stored using standard methods. Nevertheless, the general trends in the temporal profiles resulting from those samples are nearly identical to those of the current study. Specifically, all four assays detected a substantial increase in GC metabolites in the first measured samples after the health examination (in this case showing a maximum time lag of 3.5 and $4.5 \mathrm{~h}$ in the male and female, respectively). The primary difference between these measures and the more reliable measures from the 2010 experiment was the apparent sensitivity of the four assays; although the CCST assay again showed the largest increase relative to baseline values for both individuals, the CORT assay showed a larger increase than did either of the group-specific assays in the thawed samples. However, although these thawed samples provide additional evidence for the general validity of the four assays, it would be unwise to use them to compare the relative sensitivities of the assays because the different metabolites measured with each assay may vary in their stability when stored in this unconventional manner (see the differences between the metabolites measured with each of the CCST and $3 \alpha, 11 \beta$-dihydroxy-CM assays after 6 mo in the current study). Nevertheless, the strong similarities between the four sets of samples from three individual capuchins suggest that the observed patterns are likely typical of tufted capuchins.

The results of this study provide some guidelines for the analysis and interpretation of fecal GC metabolites in tufted capuchins, which we hope will facilitate field and captive work with this species, including noninvasive assessment of the welfare of laboratory- and zoo-housed capuchins. Additional considerations beyond those examined here, including seasonal variation and diurnal variation based on circadian rhythms (Palme 2005), should also be investigated, and it would be ideal to test for storage effects in samples stored at ambient temperatures (Kalbitzer and Heistermann 2013; Shutt et al. 2012). It should also be reemphasized that the results may have 
little implication for analysis of fecal GC metabolites in other species, even those closely related to tufted capuchins (Heistermann et al. 2006). Finally, it is still unknown if the CORT or $3 \alpha, 11$-oxo-CM assays provide reliable estimates of adrenocortical activity in samples extracted according to the field method and stored long term. Studies using these assays or storing samples for longer periods should ideally test these methods to ensure their validity.

Acknowledgments This study was funded in part by a Captive Care Grant from the International Primatological Society and an International Research Fellowship from the National Science Foundation (IRFP 965074) to B. C. Wheeler. We thank Francesco Natale and the keepers at the ISTC primate laboratory, Massimiliano Bianchi and Simone Catarinacci, for their logistical support. We also thank the Fondazione Bioparco and Roma Capitale-Museo Civico di Zoologia for hosting the ISTC-CNR Unit of Cognitive Primatology and Primate Center. Eugenia Polizzi di Sorrentino, Luciana Massaro, and Marialba Ventricelli provided assistance in the collection of fecal samples. We thank Dr. Fabio Faiola for providing veterinary approval and administering the ACTH challenge during the annual health examination. We are also grateful to Andrea Heistermann for performing all assays and the HPLC analyses. James Higham, Joanna Setchell, and an anonymous reviewer provided helpful comments on the manuscript.

Open Access This article is distributed under the terms of the Creative Commons Attribution License which permits any use, distribution, and reproduction in any medium, provided the original author(s) and the source are credited.

\section{References}

Addessi, E., Crescimbene, L., \& Visalberghi, E. (2007). Do capuchin monkeys (Cebus apella) use tokens as symbols? Proceedings of the Royal Society of London B: Biological Sciences, 274, 2579-2585.

Anestis, S. F. (2010). Hormones and social behavior in primates. Evolutionary Anthropology, 19, 66-78.

Bahr, N. I., Palme, R., Möhle, U., Hodges, J. K., \& Heistermann, M. (2000). Comparative aspects of the metabolism and excretion of cortisol in three individual nonhuman primates. General and Comparative Endocrinology, 117, 427-438.

Beehner, J. C., \& Whitten, P. L. (2004). Modifications of a field method for fecal steroid analysis in baboons. Physiology and Behavior, 82, 269-277.

Boinski, S., Gross, T. S., \& Davis, J. K. (1999a). Terrestrial predator alarm vocalizations are a valid monitor of stress in captive brown capuchins (Cebus apella). Zoo Biology, 18, 295-312.

Boinski, S., Swing, S. P., Gross, T. S., \& Davis, J. K. (1999b). Environmental enrichment of brown capuchins (Cebus apella): Behavioral and plasma and fecal cortisol measures of effectiveness. American Journal of Primatology, 48, 49-68.

Brown, J. L., Wemmer, C. M., \& Lehnhardt, J. (2005). Urinary cortisol analysis for monitoring adrenal activity in elephants. Zoo Biology, 14, 533-542.

Brownie, A. C. (1992). The metabolism of adrenal cortical steroids. In W. H. T. James (Ed.), The adrenal gland (pp. 209-224). New York: Raven Press.

Carosi, M., Heistermann, M., \& Visalberghi, E. (1999). Display of proceptive behaviors in relation to urinary and fecal progestin levels over the ovarian cycle in female tufted capuchin monkeys. Hormones and Behavior, 36, 252-265.

Caton, J., Hill, D., Hume, I., \& Crook, G. (1996). The digestive strategy of the common marmoset, Callithrix jacchus. Comparative Biochemistry and Physiology A: Physiology, 114, 1-8.

Charlton, B. D., Keating, J. L., Rengui, L., Huang, Y., \& Swaisgood, R. R. (2010). Female giant panda (Ailuropoda melanoleuca) chirps advertise the caller's fertile phase. Proceedings of the Royal Society of London B: Biological Sciences, 277, 1101-1106.

Colby, H., \& Kitay, J. (1972). Sex and substrate effects on hepatic corticosteroid metabolism in the rat. Endocrinology, 90, 473.

Crockford, C., Wittig, R., Langergraber, K., Ziegler, T., Zuberbühler, K., \& Deschner, T. (2013). Urinary oxytocin and social bonding in related and unrelated wild chimpanzees. Proceedings of the Royal Society of London B: Biological Sciences, 280 
Edwards, K. L., Walker, S. L., Bodenham, R. F., Ritchie, H., \& Shultz, S. (2013). Associations between social behaviour and adrenal activity in female Barbary macaques: Consequences of study design. General and Comparative Endocrinology 186, 72-79.

Engelhardt, A., Hodges, J. K., Niemitz, C., \& Heistermann, M. (2005). Female sexual behavior, but not sex skin swelling, reliably indicates the timing of the fertile phase in wild long-tailed macaques (Macaca fascicularis). Hormones and Behavior, 47, 195-204.

Fichtel, C., Kraus, C., Ganswindt, A., \& Heistermann, M. (2007). Influence of reproductive season and rank on fecal glucocorticoid levels in free-ranging male Verreaux's sifakas (Propithecus verreauxi). Hormones and Behavior, 51, 640-648.

Fragaszy, D. M., Visalberghi, E., \& Fedigan, L. M. (2004). The complete capuchin: The biology of the genus Cebus. Cambridge: Cambridge University Press.

Ganswindt, A., Palme, R., Heistermann, M., Borragan, S., \& Hodges, J. (2003). Non-invasive assessment of adrenocortical function in the male African elephant (Loxodonta africana) and its relation to musth. General and Comparative Endocrinology, 134, 156-166.

Goymann, W. (2012). On the use of non-invasive hormone research in uncontrolled, natural environments: The problem with sex, diet, metabolic rate and the individual. Methods in Ecology and Evolution, 3, $757-765$.

Groves, C. P. (2001). Primate taxonomy. Washington: Smithsonian Institution Press.

Heintz, M. R., Santymire, R. M., Parr, L. A., \& Lonsdorf, E. V. (2011). Validation of a cortisol enzyme immunoassay and characterization of salivary cortisol circadian rhythm in chimpanzees (Pan troglodytes). American Journal of Primatology, 73, 903-908.

Heistermann, M., Ademmer, C., \& Kaumanns, W. (2004). Ovarian cycle and effect of social changes on adrenal and ovarian function in Pygathrix nemaeus. International Journal of Primatology, 25, 689708.

Heistermann, M., Finke, M., \& Hodges, J. K. (1995). Assessment of female reproductive status in captivehoused Hanuman langurs (Presbytis entellus) by measurement of urinary and fecal steroid excretion patterns. American Journal of Primatology, 37, 275-284.

Heistermann, M., Palme, R., \& Ganswindt, A. (2006). Comparison of different enzymeimmunoassays for assessment of adrenocortical activity in primates based on fecal analysis. American Journal of Primatology, 68, 257-273.

Higham, J. P., MacLarnon, A. M., Ross, C., Heistermann, M., \& Semple, S. (2008). Baboon sexual swellings: Information content of size and color. Hormones and Behavior, 53, 452-462.

Hodges, J. K., \& Heistermann, M. (2011). Field endocrinology: Monitoring hormonal changes in freeranging primates. In J. M. Setchell \& D. J. Curtis (Eds.), Field and laboratory methods in primatology: A practical guide (pp. 353-370). Cambridge: Cambridge University Press.

Hunt, K. E., \& Wasser, S. K. (2003). Effect of long-term preservation methods on fecal glucocorticoid concentrations of grizzly bear and African elephant. Physiological and Biochemical Zoology, 76, 918928.

Kalbitzer, U., \& Heistermann, M. (2013). Long-term storage effects in steroid metabolite extracts from baboon (Papio sp.) faeces - a comparison of three commonly applied storage methods. Methods in Ecology \& Evolution, 4, 493-500.

Karasov, W. H., Petrossian, E., Rosenberg, L., \& Diamond, J. M. (1986). How do food passage rate and assimilation differ between herbivorous lizards and nonruminant mammals? Journal of Comparative Physiology B: Biochemical, Systemic, and Environmental Physiology, 156, 599-609.

Keay, J. M., Singh, J., Gaunt, M. C., \& Kaur, T. (2006). Fecal glucocorticoids and their metabolites as indicators of stress in various mammalian species: A literature review. Journal of Zoo and Wildlife Medicine, 37, 234-244.

Khan, M., Altmann, J., Isani, S., \& Yu, J. (2002). A matter of time: Evaluating the storage of fecal samples for steroid analysis. General and Comparative Endocrinology, 128, 57-64.

Lambert, J. E. (1998). Primate digestion: Interactions among anatomy, physiology, and feeding ecology. Evolutionary Anthropology, 7, 8-20.

Lynch, J. W., Ziegler, T. E., \& Strier, K. B. (2002). Individual and seasonal variation in fecal testosterone and cortisol levels of wild male tufted capuchin monkeys, Cebus apella nigritus. Hormones and Behavior, 41, 275-287.

Lynch Alfaro, J. W., Silva, J. D. S. E., \& Rylands, A. B. (2012). How different are robust and gracile capuchin monkeys? An argument for the use of Sapajus and Cebus. American Journal of Primatology, 74, 273-286.

Martin, R. D. (1990). Primate origins and evolution: A phylogenetic reconstruction. Princeton: Princeton University Press. 
Martínez-Mota, R., Valdespino, C., Rebolledo, J. A. R., \& Palme, R. (2008). Determination of fecal glucocorticoid metabolites to evaluate stress response in Alouatta pigra. International Journal of Primatology, 29, 1365-1373.

Milton, K. (1984). The role of food-processing factors in primate food choice. In P. S. Rodman \& J. Cant (Eds.), Adaptations for foraging in nonhuman primates (pp. 249-279). New York: Columbia University Press.

Möhle, U., Heistermann, M., Palme, R., \& Hodges, J. K. (2002). Characterization of urinary and fecal metabolites of testosterone and their measurement for assessing gonadal endocrine function in male nonhuman primates. General and Comparative Endocrinology, 129, 135-145.

Möstl, E., Maggs, J., Schrötter, G., Besenfelder, U., \& Palme, R. (2002). Measurement of cortisol metabolites in faeces of ruminants. Veterinary Research Communications, 26, 127-139.

Möstl, E., Messmann, S., Bagu, E., Robia, C., \& Palme, R. (1999). Measurement of glucocorticoid metabolite concentrations in faeces of domestic livestock. Journal of Veterinary Medicine A: Physiology Pathology Clinical Medicine, 46, 621-631.

Ostner, J., Heistermann, M., \& Schülke, O. (2008). Dominance, aggression and physiological stress in wild male Assamese macaques (Macaca assamensis). Hormones and Behavior, 54, 613-619.

Palme, R. (2005). Measuring fecal steroids: Guidelines for practical application. Annals of the New York Academy of Sciences, 1046, 75-80.

Palme, R., Fischer, P., Schildorfer, H., \& Ismail, M. (1996). Excretion of infused ${ }^{14} \mathrm{C}$-steroid hormones via faeces and urine in domestic livestock. Animal Reproduction Science, 43, 43-63.

Palme, R., \& Möstl, E. (1997). Measurement of cortisol metabolites in faeces of sheep as a parameter of cortisol concentration in blood. International Journal of Mammalian Biology, 62, 192-197.

Palme, R., Rettenbacher, S., Touma, C., El-Bahr, S., \& Möstl, E. (2005). Stress hormones in mammals and birds: Comparative aspects regarding metabolism, excretion, and noninvasive measurement in fecal samples. Annals of the New York Academy of Sciences, 1040, 162-171.

Pappano, D. J., Roberts, E. K., \& Beehner, J. C. (2010). Testing extraction and storage parameters for a fecal hormone method. American Journal of Primatology, 72, 934-941.

Pettitt, B. A., Wheaton, C. J., \& Waterman, J. M. (2007). Effects of storage treatment on fecal steroid hormone concentrations of a rodent, the Cape ground squirrel (Xerus inauris). General and Comparative Endocrinology, 150, 1-11.

Pirovino, M., Heistermann, M., Zimmermann, N., Zingg, R., Clauss, M., Codron, D., Kaup, F. J., \& Steinmetz, H. W. (2011). Fecal glucocorticoid measurements and their relation to rearing, behavior, and environmental factors in the population of pileated gibbons (Hylobates pileatus) held in European zoos. International Journal of Primatology, 32, 1161-1178.

Sabbatini, G., Vizioli, A. D. B., Visalberghi, E., \& Schino, G. (2012). Food transfers in capuchin monkeys: An experiment on partner choice. Biology Letters, 8, 757-759.

Sapolsky, R. M. (2002). Endocrinology of the stress-response. In J. Becker, S. Breedlove, D. Crews, \& M. McCarthy (Eds.), Behavioral endocrinology (2nd ed., pp. 409-450). Cambridge: MIT Press.

Schatz, S., \& Palme, R. (2001). Measurement of faecal cortisol metabolites in cats and dogs: A noninvasive method for evaluating adrenocortical function. Veterinary Research Communications, 25, 271-287.

Schwarzenberger, F., Möstl, E., Palme, R., \& Bamberg, E. (1996). Faecal steroid analysis for non-invasive monitoring of reproductive status in farm, wild and zoo animals. Animal Reproduction Science, 42, $515-526$.

Shutt, K., Setchell, J. M., \& Heistermann, M. (2012). Non-invasive monitoring of physiological stress in the western lowland gorilla (Gorilla gorilla gorilla): Validation of a fecal glucocorticoid assay and methods for practical application in the field. General and Comparative Endocrinology, 179, 167-177.

Surbeck, M., Deschner, T., Weltring, A., \& Hohmann, G. (2012). Social correlates of variation in urinary cortisol in wild male bonobos (Pan paniscus). Hormones and Behavior, 62, 27-35.

Touma, C., \& Palme, R. (2005). Measuring fecal glucocorticoid metabolites in mammals and birds: The importance of validation. Annals of the New York Academy of Sciences, 1046, 54-74.

Touma, C., Sachser, N., Möstl, E., \& Palme, R. (2003). Effects of sex and time of day on metabolism and excretion of corticosterone in urine and feces of mice. General and Comparative Endocrinology, 130, $267-278$.

Ulyan, M. J., Burrows, A. E., Buzzell, C. A., Raghanti, M. A., Marcinkiewicz, J. L., \& Phillips, K. A. (2006). The effects of predictable and unpredictable feeding schedules on the behavior and physiology of captive brown capuchins (Cebus apella). Applied Animal Behaviour Science, 101, 154-160.

von der Ohe, C. G., \& Servheen, C. (2002). Measuring stress in mammals using fecal glucocorticoids: Opportunities and challenges. Wildlife Society Bulletin, 20, 1215-1225. 
Wasser, S. K., Hunt, K. E., Brown, J. L., Cooper, K., Crockett, C. M., Bechert, U., Millspaugh, J. J., Larson, S., \& Monfort, S. L. (2000). A generalized fecal glucocorticoid assay for use in a diverse array of nondomestic mammalian and avian species. General and Comparative Endocrinology, 120, 260-275.

Wehncke, E. V., Hubbell, S. P., Foster, R. B., \& Dalling, J. W. (2003). Seed dispersal patterns produced by white-faced monkeys: Implications for the dispersal limitation of Neotropical tree species. Journal of Ecology, 91, 677-685.

Weingrill, T., Willems, E. P., Zimmermann, N., Steinmetz, H., \& Heistermann, M. (2011). Species-specific patterns in fecal glucocorticoid and androgen levels in zoo-living orangutans (Pongo spp.). General and Comparative Endocrinology, 172, 446-457.

Whitten, P. L., Brockman, D. K., \& Stavisky, R. C. (1998a). Recent advances in noninvasive techniques to monitor hormone-behavior interactions. Yearbook of Physical Anthropology, 41, 1-23.

Whitten, P. L., Stavisky, R., Aureli, F., \& Russell, E. (1998b). Response of fecal cortisol to stress in captive chimpanzees (Pan troglodytes). American Journal of Primatology, 44, 57-69.

Young, K., Walker, S., Lanthier, C., Waddell, W., Monfort, S. L., \& Brown, J. L. (2004). Noninvasive monitoring of adrenocortical activity in carnivores by fecal glucocorticoid analyses. General and Comparative Endocrinology, 137, 148-165.

Ziegler, T. E., \& Wittwer, D. J. (2005). Fecal steroid research in the field and laboratory: improved methods for storage, transport, processing, and analysis. American Journal of Primatology, 67, 159-174. 